\title{
Primer design and amplification efficiencies are crucial for reliability of quantitative PCR studies of caffeine biosynthetic $\mathrm{N}$-methyltransferases in coffee
}

\author{
Simmi P. Sreedharan ${ }^{1} \cdot$ Avinash Kumar $^{1} \cdot$ Parvatam Giridhar $^{1}$
}

Received: 7 August 2018 / Accepted: 19 October 2018 / Published online: 1 November 2018

(c) The Author(s) 2018

\begin{abstract}
Primers having suboptimal amplification efficiencies were shown to falsely represent fold change expression of the $N$-methyltransferases gene family involved in caffeine biosynthesis in Coffea canephora. To study this phenomenon, the role of stability of the internal reference gene, as well as the amplification efficiency correction of the primers was investigated. GAPDH and Ubiquitin exhibited a good stability for studying the ontogeny of endosperm tissue, as well as the leaf transcriptome during stress from salicylic acid, methyl jasmonate, PEG-mediated drought and sudden exposure to light. Ubiquitin manifested low variation in Cq under all these stress regimes and in endosperm ontogeny with 30.1-30.9 in the best dataset and 28.8-30.9 in the most deviating dataset. It was observed that problems arising due to improper amplification efficiency of the target or reference genes or both could lead to misinterpretation of gene expression levels. Quantitative RT-PCR performed at a suboptimal efficiency of GAPDH reference gene at 1.68 led to the faulty interpretation of 2.007 folds upregulation by the $2^{-\Delta \Delta C t}$ method and 1.705 folds upregulation by Efficiency method for the first NMT (Xanthosine methyltransferase), which actually is repressed during dark acclimatization of coffee plants. Efficiency correction improved the reliability of the expression data and also indicated a downregulation of this gene by 0.485 folds and 0.474 folds using $2^{-\Delta \Delta C t}$ and $\mathrm{E}$ method, respectively, in concordance to earlier reports. Hence, efficiency correction of the primers having suboptimal efficiencies is an absolute prerequisite for the accurate calculation of fold change using quantitative RT-PCR.
\end{abstract}

Keywords Abiotic stress $\cdot$ Caffeine $\cdot$ Internal reference gene $\cdot$ Normalization

\section{Introduction}

Caffeine is the most researched molecule from coffee and is designated to a functional role under the 'Chemical Defense Theory' (Frischknecht et al. 1985). Nevertheless, there has been much debate about the actual role and the evolution of caffeine in different plant systems. Caffeine accounts for the major purine alkaloid in coffee and is synthesized from the ubiquitous committed precursor, xanthosine, by the Salicylic Acid Benzoic Acid THeobromine Synthase, (SABATH) superfamily of methyltransferases (Kato and Mizuno 2004). With the availability of large EST database and complete

Simmi P. Sreedharan and Avinash Kumar Equal Contribution.

Parvatam Giridhar

parvatamg@yahoo.com

$1 \quad$ Plant Cell Biotechnology Department, CSIR-Central Food Technological Research Institute, Mysuru, Karnataka, India sequencing of C. canephora genome (Denouend et al. 2014) much research needs to now focus on transcriptomics and functional characterization of the coffee genome. Caffeine biosynthetic $N$ - methyltransferases are upregulated under influence from light (Kumar et al. 2015a), developmental stage and genotype (Perrios et al. 2015), salicylic acid and methyl jasmonate (Kumar et al. 2015b, 2017). In addition, salinity and drought negatively regulate caffeine content (Kumar et al. 2015c). Future studies on the regulation of caffeine biosynthesis require the assignment of a suitable reference gene for normalization of quantitative PCR.

Quantitative RT-PCR remains a popular tool for transcriptomics due to its ease of application and economic feasibility. Search for appropriate reference gene is indispensable for normalization of qPCR quantification (Kozera and Rapacz 2013). Housekeeping genes like actin, tubulin, GAPDH, ubiquitin, rpl39, and rRNA are routinely used as internal reference genes in different systems (Joseph et al. 2018). However, the reference genes itself vary in their 
stability and expression level under certain biological conditions. Minimum Information for publication of Quantitative real-time PCR Experiments (MIQE) guidelines (Bustin 2009) recommends the use of most stable reference gene or their combinations for the quantitative analysis of the experimental sets. Technically, it is not feasible to identify a single reference control that can be used for all the experimental sets. Nevertheless, using the statistical programs most suitable combination of reference genes can be selected for each biological experiment sets. The most popular algorithms used for identifying stability of the reference genes include GeNorm (Vandesompele et al. 2002), NormFinder (Andersen et al. 2004), BestKeeper (Pfaffl et al. 2004) and comparative delta-CT (Silver et al. 2006) method, all of which provide a value of stability or normalization factor based on expression of the reference gene in different test and control samples.

Conceptually, relative gene expression by qPCR is calculated by the efficiency method (E method) (Pfaffl 2001), which account for the differences in PCR efficiency of the internal reference gene and target gene and $2^{-\Delta \Delta \mathrm{Ct}}$ method that assumes $100 \%$ efficiency for both target and reference (Livak 2001). PCR efficiency between samples varies due to dissimilarities in the quantity and quality of cDNA, primer quality, the copy number of transcripts and annealing temperatures. Suboptimal quality of template and primer contribute to errors in calculated fold change due to an appearance of non-stochastic $\mathrm{Cq}$ values in the standard curve (Ruijter et al. 2012). PCR efficiency is a critical indicator for the performance of qPCR analysis of multigene families especially involved in secondary metabolism (Arunraj and Samuel 2018). Specificities of primers are not always guaranteed while working with multigene families and the quality of template may vary between samples depending on the impurities in prepared template due to changes in secondary metabolism. Hence, neither $2^{-\Delta \Delta C T}$ method nor Pfaffl's E method are necessarily the most accurate description of the actual fold change until efficiencies are optimally corrected. Large-scale qPCR analysis of multigene families requires proper optimization considering all these factors for the generation of reliable data. The present study establishes such errors arising in the quantitation of caffeine biosynthetic NMT gene family in Coffee under light stress and the effect of efficiency correction on the reliability of expression data.

\section{Materials and methods}

\section{Plant materials and sample preparation}

Total RNA was isolated from Coffea canephora Pierre ex. Froehner var. robusta cv. S274 leaves of stress treatments (Kumar et al. 2015a, b, 2017) and developing endosperms
(Giridhar et al. 2012) and all the primers were adopted from the same studies. Only plant samples from $50 \mu \mathrm{M}$ salicylic acid, $10 \mu \mathrm{l}$ methyl jasmonate, $200 \mathrm{mM}$ sodium chloride and $15 \%(\mathrm{w} / \mathrm{v})$ polyethylene glycol treatments were considered for the present study. Light and endosperm sampling was exactly similar to the method used previously (Kumar et al. 2015a, b). First-strand cDNA synthesis with $1 \mu \mathrm{g}$ of total RNA was carried out using iScript cDNA synthesis kit (BioRad) with pre-mixed cocktail of Oligo-dT and random hexamer primers. The cDNA preparations from circadian undisturbed and dark acclimatized samples alone were used for the study of fold change.

\section{Test of stability of internal reference}

Stability of the reference genes, GAPDH and Ubiquitin, were carried out by comparing $\mathrm{Cq}$ values in control and treated samples of stressed plants and in the ten different growth phases of endosperms. Twofold dilutions of control leaf and endosperm cDNA (1, 1:2, 1:4, 1:8, 1:16, 1:32 and 1:64 dilutions) was used to generate standard plots of the reference genes and the $\mathrm{Cq}$ was corrected using the formula $\mathrm{c} 2=\mathrm{c} 1 * \log (\mathrm{a} 1) / \log (\mathrm{a} 2)$ where $\mathrm{c} 2$ is the corrected Cq value, if the sample would have amplified with the efficiency equaling a2. For leaf samples, the Cq of Ubiquitin was corrected according to the efficiency of $G A P D H$ and in ontogeny the Cq of GAPDH was corrected according to that of Ubiquitin. Amplification curves obtained for the experimental setup using 1:15 diluted cDNA as a template was analyzed using RefFinder program (http://leonxie.esy.es/RefFinder/). qPCR was performed in Applied Biosystems Quantstudio5 instrument using Ssofast Evagreen master mix (BioRad) supplemented with ROXII (Takara biosciences) with following parameters: initial denaturation of $95^{\circ} \mathrm{C}$ for $30 \mathrm{~s}$, followed by 40 cycles of denaturation at $95{ }^{\circ} \mathrm{C}$ for $5 \mathrm{~s}$ and annealing/ extension at $\left(58{ }^{\circ} \mathrm{C}\right)$ for $30 \mathrm{~s}$.

\section{Standard curve for efficiency calculation}

Standard plot was made from a dilution series of 1:5, 1:10, 1:20 and 1:40 dilutions of mixed cDNA pooled from all the stress samples. Reactions were repeated in three different annealing temperatures $\left(55^{\circ} \mathrm{C}, 57^{\circ} \mathrm{C}\right.$ and $\left.59^{\circ} \mathrm{C}\right)$. qPCR reactions were carried out in Applied Biosystems Quantstudio 5 instrument with Ssofast Evagreen master mix (BioRad) supplemented with ROXII (Takara biosciences). The cycle parameters included initial denaturation of $95{ }^{\circ} \mathrm{C}$ for $30 \mathrm{~s}$, cycle denaturation at $95{ }^{\circ} \mathrm{C}$ for $5 \mathrm{~s}$ and annealing/extension at $\left(55-59^{\circ} \mathrm{C}\right)$ for $30 \mathrm{~s}$ ( 40 cycle). Efficiency of reaction was calculated from the slope using the formula $E=10^{-1 / \text { slope }}$. Fold change between cDNA of circadian undisturbed and dark acclimatized plant samples for XMT and MXMT genes was calculated using the method of Pfaffl (2001), 
ratio $=\frac{\left(E_{\text {target }}\right)^{\Delta \mathrm{C} \text { target (control-target) }}}{\left(E_{\text {ref }}\right)^{\Delta \mathrm{CP}_{\text {ref }} \text { (Control-target) }}}$ and $2^{-\Delta \Delta \mathrm{Ct}}$ method (Livak 2001).

\section{Results and discussion}

\section{Stability of Glyceraldehyde 3-phosphate dehydrogenase (GAPDH) and Ubiquitin internal reference genes}

Most suitable internal reference gene for qPCR by SYBR chemistry has been identified for coffee to study rust infection (Vieira et al. 2011), coffee berry disease (Figueiredo et al. 2013), embryogenic tissue (Freitas et al. 2017), water stress (Fernandes-Brum et al. 2017) and chilling stress (Goulao et al. 2011). GAPDH and Ubiquitin are considered as the most stable internal reference genes combination for qPCR expression studies during water stress and GAPDH for studies on fruit ontogeny (Barsalobres-Cavallari et al. 2009; Cruz et al. 2009). In the present study, the stability of both the internal reference gene was tested under conditions of light exposure, salicylic acid, methyl jasmonate, salinity and drought stress on leaf cDNA and during development in endosperm tissues. A box plot graph indicates the higher power of Ubiquitin as a reference gene in the analysis between control and stress treated samples from leaves of salicylic acid, methyl jasmonate, PEG-mediated drought and light exposed plants as well as in developing endosperms (Fig. 1a, b). Neither GAPDH nor Ubiquitin were suitable for studying salinity stress. Strictly essential genes like EF1 and $E F 1 \alpha$ were predicted to be suitable reference genes for salinity stress in another study on C. arabica (Carvalho et al. 2013). The frequencies of Cq values for control and treated samples of leaf and for developing endosperms distributed normally (Fig. 1c, d). Standard curves also indicated that Ubiquitin has more power in studying the difference in gene expression between leaf and endosperm tissues (Fig. 1e, f). The amplification curves could be most suitably used at dilutions of 1:4 to 1:32 for both GAPDH and Ubiquitin.

Stability values were obtained for GAPDH and Ubiquitin in intra and inter-group comparisons using comparative delta CT, Bestkeeper, NormFinder and GeNorm encompassed in the RefFinder package. The data described in Table 1 indicated that stability values improve by use of efficiency corrected Cq. This difference in efficiency could affect the range of fold change while using these two reference genes or while comparing a reference with the target. M-value of the stress subset was 0.760 in GeNorm analysis of the uncorrected $\mathrm{Cq}$ values. However, the $\mathrm{M}$-value improved to 0.039 after efficiency correction of $\mathrm{Cq}$ indicating $G A P D H$ and Ubiquitin to be a stable combination of reference for fold change calculation involving stress experiments. Addition of the endosperm dataset to the consolidated data, disrupted the stability value $(M=0.856$ for uncorrected and $M=3.240$ for corrected). Stability values for GAPDH and Ubiquitin using NormFinder was lowest for salicylic acid treatment (0.009) and highest for endosperm ontogeny (0.053). RefFinder analysis also indicated that the average standard deviation with delta CT method reduced upon Cq correction in individual subsets as well as 'treatment alone' consolidated data, whereas, an opposite was observed on the total combined stress and endosperm dataset. Moreover, the uncorrected $\mathrm{Cq}$ values for certain subsets varied at $>1.0$ values. Hence, $2^{-\Delta \Delta \mathrm{CT}}$ would not be an appropriate method to calculate fold change using these reference genes. Also, correcting $\mathrm{Cq}$ for reference genes reduced the standard deviations of $\Delta \mathrm{Ct}$ observed for different datasets.

\section{Effect of efficiency correction on data analysis of fold change}

Quantitative RT-PCR analysis of transcripts of multigene families, especially genes involved in secondary metabolism, suffer due to issues relating to the specificity of primer and purity of isolated RNA. C. canephora NMTs share greater than $80 \%$ similarity (Denoued et al. 2014), an example of which is depicted in Fig. 2a. The primers used in this study were designed based on EST sequences prior to the publication of the coffee genome (Fig. 2b). Though the primers amplified specific genes, the CCMXMTI forward and $C c D X M T$ reverse primer has a base substitution at 10th 124 and 18th /24 nucleotide position from the $5^{\prime}$ end of the primer, respectively. Also, the $M X M T$ reverse primer shows cross specificity to $C C M T L 1$ and the $C C D X M T$ reverse primer to $C c M T L$ and $C c M X M T$ - both of which could effectively alter their PCR efficiencies and its dynamics under different experimental datasets. Additionally, the primer binding site on the genome are slightly prone to contain SNPs and hence may have single mismatches depending of genotypes. Also, the primer pairs had varying amplification efficiencies at different temperatures. For $G A P D H$, efficiencies are 1.68 $\left(55^{\circ} \mathrm{C}\right), 2.01\left(57^{\circ} \mathrm{C}\right)$ and $1.84\left(59^{\circ} \mathrm{C}\right)$. Ubiquitin amplified with more consistent efficiencies of $2.01,1.98,1.98$, respectively at these temperatures. XMT primers amplified at efficiencies of $1.93\left(55^{\circ} \mathrm{C}\right)$ and $2.10\left(57^{\circ} \mathrm{C}\right)$ whereas, MXMT primers at 1.68 and 1.97 and DXMT primers at 1.96 and 2.39, respectively. MXMT1 primers worked only at $55{ }^{\circ} \mathrm{C}$ with 1.67 efficiency. It was noted that primers that had optimal design according to Coffee genomic sequence, for example, $X M T$ and Ubiquitin, did not vary much in efficiency at different annealing temperatures. However, the sub-optimally designed GAPDH and MXMT exhibited

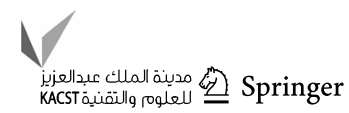


a

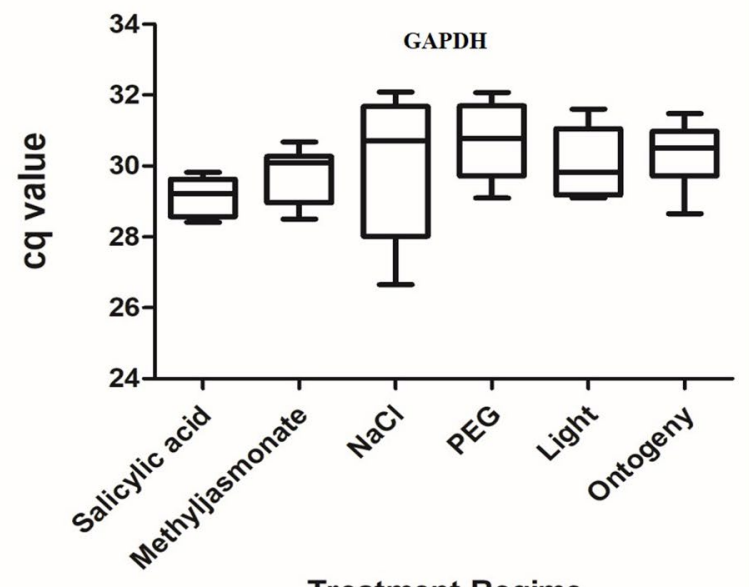

C
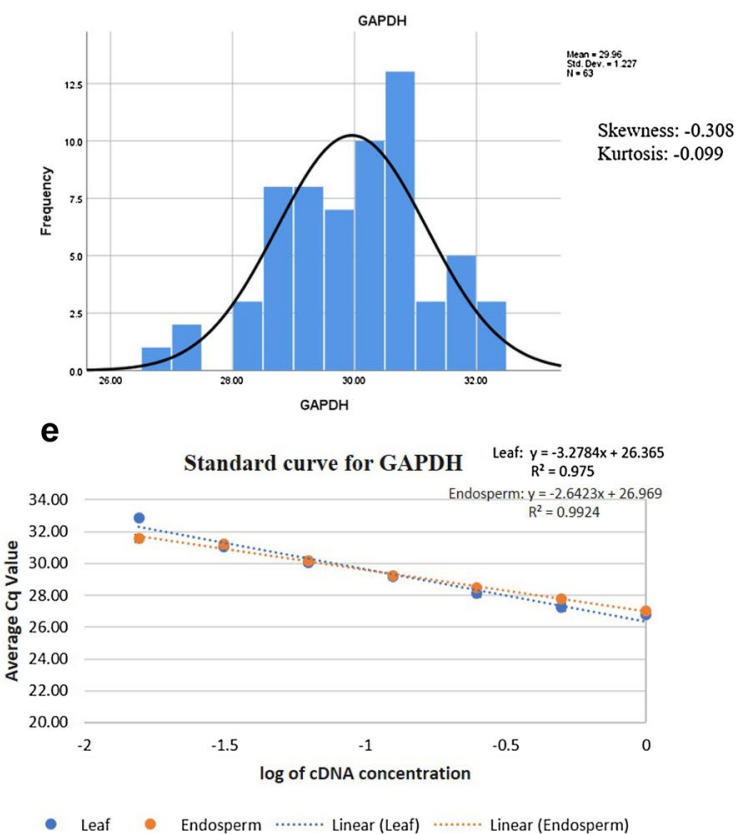

Fig. 1 Variation in internal reference genes and their standard curves. Box plot depicting the variation of Cq of (a) GAPDH and (b) Ubiquitin under different experimental conditions of abiotic stress and ontogeny in Coffea canephora; $\mathrm{Cq}$ values showing normal distribu-

higher difference in efficiency at a slight change of annealing temperature.

A minute repression of caffeine and NMT expression is observed in the leaves of dark acclimatized plants of coffee when compared to circadian undisturbed plants (Kumar et al. 2015a). Hence, these samples were used to study the effect of amplification efficiencies on fold change calculation. The calibrator DNA was prepared from pooled samples of a larger dataset as mentioned in material and methods. The fold change was calculated by the $2^{-\Delta \Delta \mathrm{Ct}}$ method (Livak

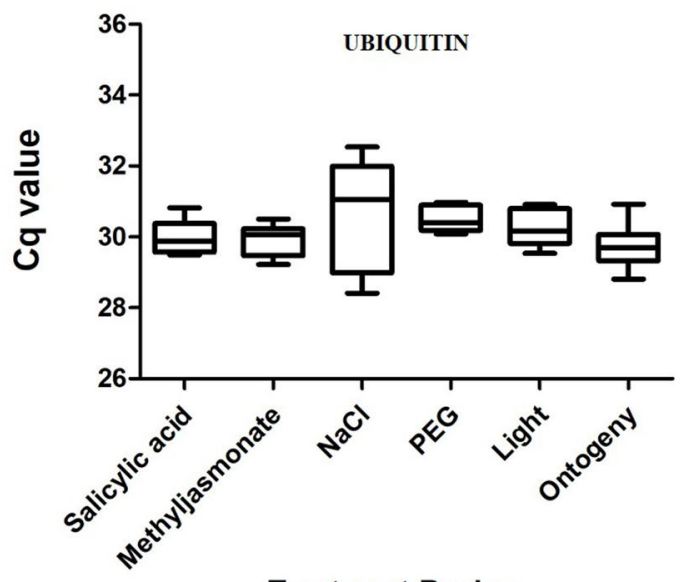

d

\section{Treatment Regime}
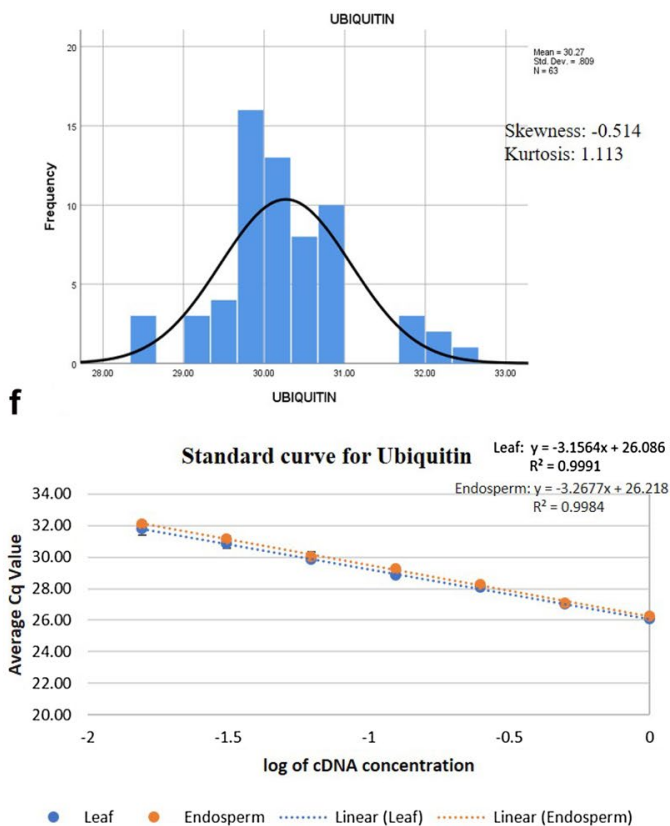

tion for (c) GAPDH and (d) Ubiquitin; standard curve plotted from different dilutions of leaf and endosperm cDNA for (e) GAPDH and (f) Ubiquitin

2001) and the E method (Pfaffl 2001), with consideration of correction under optimal PCR annealing temperatures. $X M T$ and Ubiquitin genes amplify with good efficiency at both $55^{\circ} \mathrm{C}$ and $57^{\circ} \mathrm{C}$. Fold change in XMT using Ubiquitin reference gave comparable results using $2^{-\Delta \Delta \mathrm{Ct}}$ method (0.915 fold) and $\mathrm{E}$ method ( 0.913 fold) at $55^{\circ} \mathrm{C}$ and efficiencies of 1.89 and 1.99 . Fold change calculation by efficiency correction using standard plot at annealing temperature of $57{ }^{\circ} \mathrm{C}$ was also comparable between $2^{-\Delta \Delta \mathrm{Ct}}$ method $(0.680$ fold) and E method (0.668 fold). However, at suboptimal 
Table 1 Stability of Glyceraldehyde 3-phosphate dehydrogenase and Ubiquitin reference genes in different experimental datasets

\begin{tabular}{|c|c|c|c|c|c|c|c|}
\hline \multirow[t]{2}{*}{ Group } & \multirow[t]{2}{*}{ Gene } & \multirow{2}{*}{$\begin{array}{l}\text { RefFinder com- } \\
\text { prehensive }\end{array}$} & \multirow[t]{2}{*}{ Delta Ct } & \multicolumn{2}{|c|}{ BestKeeper } & \multirow[t]{2}{*}{ NormFinder } & \multirow[t]{2}{*}{ GeNorm } \\
\hline & & & & $\mathrm{SD} \pm \mathrm{Cq}$ & $\mathrm{CV} \pm[\%]$ & & \\
\hline \multicolumn{8}{|l|}{ Efficiency corrected $\mathrm{Cq}$} \\
\hline \multirow[t]{3}{*}{ SA } & UBI & 1.00 & 0.02 & 0.47 & 1.66 & 0.009 & - \\
\hline & GAPDH & 1.68 & 0.02 & 0.48 & 1.66 & 0.009 & - \\
\hline & GAPDH/UBI & - & - & - & - & - & 0.018 \\
\hline \multirow[t]{3}{*}{ MEJ } & UBI & 1.00 & 0.02 & 0.62 & 2.19 & 0.012 & - \\
\hline & GAPDH & 1.68 & 0.02 & 0.65 & 2.19 & 0.012 & - \\
\hline & GAPDH/UBI & - & - & - & - & - & 0.025 \\
\hline \multirow[t]{3}{*}{$\mathrm{NaCl}$} & UBI & 1.00 & 0.06 & 1.27 & 4.34 & 0.029 & - \\
\hline & GAPDH & 1.68 & 0.06 & 1.31 & 4.34 & 0.029 & - \\
\hline & GAPDH/UBI & - & - & - & - & - & 0.059 \\
\hline \multirow[t]{3}{*}{ PEG } & UBI & 1.00 & 0.04 & 0.84 & 2.82 & 0.019 & - \\
\hline & GAPDH & 1.68 & 0.04 & 0.87 & 2.82 & 0.019 & - \\
\hline & GAPDH/UBI & - & - & - & - & - & 0.037 \\
\hline \multirow[t]{3}{*}{ Light } & UBI & 1.00 & 0.03 & 0.83 & 2.90 & 0.017 & - \\
\hline & GAPDH & 1.68 & 0.03 & 0.86 & 2.90 & 0.017 & - \\
\hline & GAPDH/UBI & - & - & - & - & - & 0.034 \\
\hline \multirow[t]{3}{*}{ Ontogeny } & UBI & 1.19 & 0.11 & 0.41 & 1.38 & 0.053 & - \\
\hline & GAPDH & 1.41 & 0.11 & 0.33 & 1.38 & 0.053 & - \\
\hline & GAPDH/UBI & - & - & - & - & - & 0.106 \\
\hline \multirow[t]{3}{*}{ Consolidated } & UBI & 1.00 & 3.24 & 0.83 & 2.86 & 1.620 & - \\
\hline & GAPDH & 1.68 & 3.24 & 2.69 & 9.64 & 1.620 & - \\
\hline & GAPDH/UBI & - & - & - & - & - & 3.240 \\
\hline \multirow[t]{3}{*}{ Consolidated minus ontogeny } & UBI & 1.00 & 0.04 & 0.92 & 3.19 & 0.019 & - \\
\hline & GAPDH & 1.68 & 0.04 & 0.95 & 3.19 & 0.019 & - \\
\hline & GAPDH/UBI & - & - & - & - & - & 0.039 \\
\hline \multicolumn{8}{|l|}{ Efficiency Un-corrected Cq } \\
\hline \multirow[t]{3}{*}{ SA } & UBI & 1.00 & 0.40 & 0.35 & 1.15 & 0.201 & - \\
\hline & GAPDH & 1.68 & 0.40 & 0.48 & 1.66 & 0.201 & - \\
\hline & GAPDH/UBI & - & - & - & - & - & 0.401 \\
\hline \multirow[t]{3}{*}{ MEJ } & UBI & 1.00 & 0.44 & 0.34 & 1.13 & 0.219 & - \\
\hline & GAPDH & 1.68 & 0.44 & 0.64 & 2.15 & 0.219 & - \\
\hline & GAPDH/UBI & - & - & - & - & - & 0.437 \\
\hline \multirow[t]{3}{*}{$\mathrm{NaCl}$} & UBI & 1.19 & 0.77 & 1.35 & 4.39 & 0.386 & - \\
\hline & GAPDH & 1.41 & 0.77 & 1.31 & 4.34 & 0.386 & - \\
\hline & GAPDH/UBI & - & - & - & - & - & 0.772 \\
\hline \multirow[t]{3}{*}{ PEG } & UBI & 1.00 & 0.95 & 0.33 & 1.09 & 0.475 & - \\
\hline & GAPDH & 1.68 & 0.95 & 0.87 & 2.82 & 0.475 & - \\
\hline & GAPDH/UBI & - & - & - & - & - & 0.950 \\
\hline Light & UBI & 1.00 & 0.72 & 0.41 & 1.35 & 0.359 & - \\
\hline & GAPDH & 1.68 & 0.72 & 0.86 & 2.90 & 0.359 & - \\
\hline & GAPDH/UBI & - & - & - & - & - & 0.719 \\
\hline Ontogeny & UBI & 1.00 & 0.71 & 0.41 & 1.38 & 0.353 & - \\
\hline & GAPDH & 1.68 & 0.71 & 0.65 & 2.13 & 0.353 & - \\
\hline & GAPDH/UBI & - & - & - & - & - & 0.706 \\
\hline Consolidated & UBI & 1.00 & 0.86 & 0.55 & 1.83 & 0.428 & - \\
\hline & GAPDH & 1.68 & 0.86 & 0.88 & 2.95 & 0.428 & - \\
\hline & GAPDH/UBI & - & - & - & - & - & 0.856 \\
\hline
\end{tabular}


Table 1 (continued)

\begin{tabular}{|c|c|c|c|c|c|c|c|}
\hline \multirow[t]{2}{*}{ Group } & \multirow[t]{2}{*}{ Gene } & \multirow{2}{*}{$\begin{array}{l}\text { RefFinder com- } \\
\text { prehensive }\end{array}$} & \multirow[t]{2}{*}{ Delta $\mathrm{Ct}$} & \multicolumn{2}{|c|}{ BestKeeper } & \multirow[t]{2}{*}{ NormFinder } & \multirow[t]{2}{*}{ GeNorm } \\
\hline & & & & $\mathrm{SD} \pm \mathrm{Cq}$ & $\mathrm{CV} \pm[\%]$ & & \\
\hline \multirow[t]{3}{*}{ Consolidated minus ontogeny } & UBI & 1.00 & 0.74 & 0.57 & 1.87 & 0.368 & - \\
\hline & GAPDH & 1.68 & 0.74 & 0.95 & 3.19 & 0.368 & - \\
\hline & GAPDH/UBI & - & - & - & - & - & 0.736 \\
\hline
\end{tabular}

a

CcNMT1
CcNMT2
CcNMT3
CcMXMT
CcMTL
CcXMT
CcDXMT
CcNMT15

CcNMT1 CcNMT2 CcNMT3 CCMXMT CcMTL CcXMT CcDXMT CCNMT15

(1)

\begin{tabular}{|c|c|c|c|c|c|c|c|c|}
\hline Gene Name & Primer Sequence & $\% \mathrm{GC}$ & $\begin{array}{c}\text { Max. } \\
\Delta \mathrm{G} \\
\text { Homo- } \\
\text { dimer }\end{array}$ & $\begin{array}{l}\text { Max. } \Delta G \\
\text { Hairpin }\end{array}$ & $\begin{array}{c}\text { Max. } \\
\Delta \text { G Hetero- } \\
\text { dimer }\end{array}$ & $\begin{array}{l}\text { Amplicon Size } \\
\text { (bp) }\end{array}$ & $\begin{array}{l}\text { Annealing } \\
\text { Temperature }\end{array}$ & $\begin{array}{c}\text { PCR } \\
\text { Amplification } \\
\text { Efficiency }\end{array}$ \\
\hline \multirow{2}{*}{ GAPDH } & F: S' ACG ATA GGT TTG GCA TTG T $3^{\prime}$ & $42.1 \%$ & -3.61 & 0.62 & \multirow{2}{*}{-5.37} & \multirow{2}{*}{139} & $55^{\circ} \mathrm{C}$ & 1.70 \\
\hline & R: $5^{\prime}$ GTG CTA CTG GGA ATA ATG II $3^{\prime}$ & $40 \%$ & -3.14 & 0.75 & & & $57^{\circ} \mathrm{C}$ & 2.00 \\
\hline \multirow{2}{*}{ UBI } & $\mathrm{F}: 5^{\prime}$ GGG TGG AGG AGA AAG AAG GAAT $3^{\prime}$ & $50 \%$ & $-1,47$ & 4.1 & \multirow{2}{*}{-12.61} & \multirow{2}{*}{144} & $55^{\circ} \mathrm{C}$ & 2.01 \\
\hline & R: $5^{\prime}$ CTC CAC CTC TCA GAG CAA GAAC $3^{\prime}$ & $54.5 \%$ & -4.77 & -0.07 & & & $57^{\circ} \mathrm{C}$ & 1.98 \\
\hline \multirow{2}{*}{ CCXMT } & F: 5' TGT AAA GGA GIT GAA TTA GAC GCC 3' & $41.7 \%$ & -5.36 & -0.54 & \multirow{2}{*}{-6.53} & \multirow{2}{*}{250} & $55^{\circ} \mathrm{C}$ & 1.93 \\
\hline & R: $5^{\prime}$ CTG CTT TAA TAT GIT CAT CGT CAA T $3^{\prime}$ & $32 \%$ & -4.85 & 0.95 & & & $57^{\circ} \mathrm{C}$ & 2.10 \\
\hline \multirow{2}{*}{ CoMXMT } & F: $5^{\prime}$ ATA GTT TCA ATA TTC CAT TCT TTAC $3^{\prime}$ & $24 \%$ & -7.8 & 0.52 & \multirow{2}{*}{-6.83} & \multirow{2}{*}{209} & $55^{\circ} \mathrm{C}$ & 1.68 \\
\hline & R: $5^{\prime}$ GGG TTC GTA AAC TGA TCT AAT TAA T $3^{\prime}$ & $32 \%$ & -7.8 & -1.02 & & & $57^{\circ} \mathrm{C}$ & $1.97^{*}$ \\
\hline \multirow{2}{*}{ CCMXMT-1 } & F: $5^{\prime}$ ACC CAG TAA GAT CCC ATG AACA $3^{\prime}$ & $45.5 \%$ & -5.38 & 0.57 & \multirow{2}{*}{-3.43} & \multirow{2}{*}{201} & $55^{\circ} \mathrm{C}$ & 1.67 \\
\hline & R: $5^{\prime}$ GAG AGA AAT GAT AAG ATT ATT ATA GC $3^{\prime}$ & $26.9 \%$ & -4.38 & -0.97 & & & $57^{\circ} \mathrm{C}$ & NA \\
\hline \multirow{2}{*}{ CeDXMT } & F: $5^{\prime}$ ACG TGG CCG AAT GCT CCT TAC $3^{\prime}$ & $57.1 \%$ & -9.28 & -1.56 & \multirow{2}{*}{-7.13} & \multirow{2}{*}{354} & $55^{\circ} \mathrm{C}$ & 1.96 \\
\hline & R: $5^{\prime}$ GGT TCG AAA ATT GAT CTA ACG ACA $3^{\prime}$ & $37.5 \%$ & $-10,65$ & -0.57 & & & $57^{\circ} \mathrm{C}$ & 2.39 \\
\hline
\end{tabular}

C
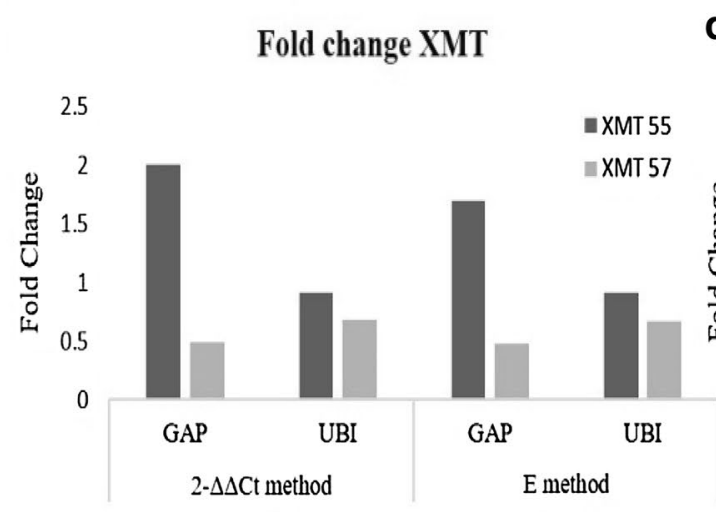

Fig. 2 Effects of primers quality and specificity on fold change of the homologous caffeine biosynthetic genes. a Sequence similarity in $C$. canephora NMTs; b properties of primers; fold change calculated d

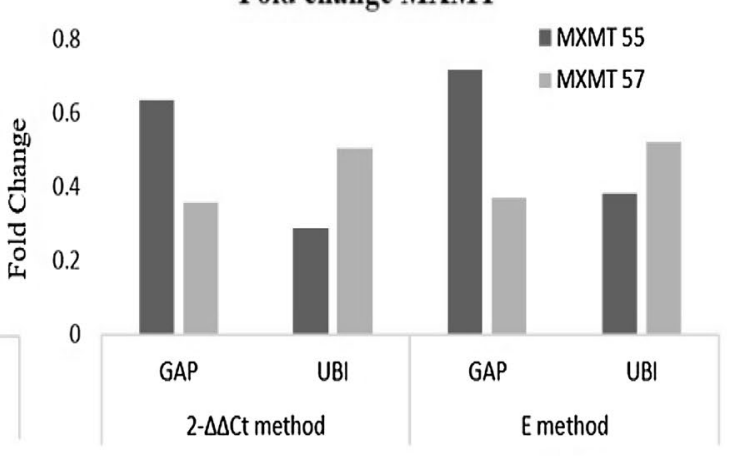

using $2^{-\Delta \Delta C T}$ method and $\mathrm{E}$ method with consistent and inconsistent efficiencies of target and reference gene, $\mathbf{c}$ fold change for XMT gene, d fold change for MXMT gene 
temperature for $G A P D H$ at $55{ }^{\circ} \mathrm{C}$, the calculated fold change was different between $2^{-\Delta \Delta \mathrm{Ct}}$ method (2.007 fold) and E method (1.705 fold). Furthermore, it is known that the XMTs are downregulated when circadian undisturbed plants are subjected to dark conditions (Kumar et al. 2015a). It is interpreted that at suboptimal efficiencies of reference gene, neither the $2^{-\Delta \Delta \mathrm{Ct}}$ method nor $E$ method were able to correct an erroneous upregulation plotted for XMT target gene. However, the efficiency of GAPDH and XMT corrected by standard curve plot at $57{ }^{\circ} \mathrm{C}$, markedly reduced the fold change to 0.485 folds and 0.474 folds by $2^{-\Delta \Delta \mathrm{Ct}}$ and $E$ method, respectively. Upon correction of efficiency, the fold change calculated for XMT with GAPDH became comparable to the fold change calculated using Ubiquitin.

MXMT amplification is prone to relaxed specificity from the reverse primer and also the primer set work at low efficiency at $55^{\circ} \mathrm{C}$ as similar to GAPDH. Performing efficiency corrections at $57^{\circ} \mathrm{C}$ led to more consistency in fold change comparisons using the different methods of $2^{-\Delta \Delta \mathrm{Ct}}$ and $E$ method as for XMT. However, the dynamics of fold change were different when comparing between different reference genes.

\section{Conclusions}

GAPDH and Ubiquitin genes have low variability between control samples and treatments involving salicylic acid, methyl jasmonate, light exposure, PEG and ontogeny of endosperms with correction of their efficiencies. Primer efficiency is crucial for reduction of errors in fold change calculations of caffeine biosynthetic NMTs. Efficiency correction by qPCR standardization overcomes the errors in the range of fold changes. Hence, we reiterate the importance of efficiency calculations of individual primers as inevitable prior to expression studies. In addition, this could reduce the chances of falsely interpreting overexpression for genes that may actually be repressed or to prevent exaggeration of fold changes.

Acknowledgements SPS and AK are recipient of fellowship from Council of Scientific and Industrial Research, New Delhi. Authors thank Dr. Surya Prakash Rao, Central Coffee Research Institute, Karnataka, India, for sampling of Coffee fruits and Dr. Nandini P. Shetty, CSIR-Central Food Technological Research Institute, Karnataka, India, for facilitating with qPCR equipment. The project was supported by Grants from Science and Engineering Research Board, Department of Science and Technology, New Delhi under the Grant number: SERB/ $\mathrm{SR} / \mathrm{SO} / \mathrm{PS} / 20 / 2012$.

Author contributions SPS and AK designed the experiments. SPS carried out all the qPCRs. PG supervised the work. AK and SPS analyzed the data and drafted the manuscript. All authors approve the final version of the draft.

\section{Compliance with ethical standards}

Conflict of interest The authors declare no conflicts of interest.

Open Access This article is distributed under the terms of the Creative Commons Attribution 4.0 International License (http://creativeco mmons.org/licenses/by/4.0/), which permits unrestricted use, distribution, and reproduction in any medium, provided you give appropriate credit to the original author(s) and the source, provide a link to the Creative Commons license, and indicate if changes were made.

\section{References}

Andersen CL, Jensen JL, Orntoft TF (2004) Normalization of real-time quantitative reverse transcription-PCR data: a model-based variance estimation approach to identify genes suited for normalization, applied to bladder and colon cancer data sets. Cancer Res 64:5245-5250

Arunraj R, Samuel MA (2018) Integration of amplification efficiency in qPCR analysis allows precise and relative quantification of transcript abundance of genes from large gene families using RNA isolated from difficult tissues. Brief Funct Genomics 17:147-115

Barsalobres-Cavallari CF, Severino FE, Maluf MP, Maia IG (2009) Identification of suitable internal control genes for expression studies in Coffea arabica under different experimental conditions. BMC Mol Biol 10:1-11

Bustin SA, Benes V, Garson JA, Hellemans J, Huggett J, Kubista M, Mueller R, Nolan T, Pfaffl MW, Shipley GL, Vandesompele J, Wittwer CT (2009) The MIQE guidelines: minimum information for publication of quantitative real-time PCR experiments. Clin Chem 55:611-622

Cruz F, Kalaoun S, Nobile P, Colombo C, Almeida J, Barros MG, Romano E, Grossi-de-Sa MF et al (2009) Evaluation of coffee reference genes for relative expression studies by quantitative realtime RT-PCR. Mol Breed 23:607-616

de Carvalho K, Bespalhok Filho JC, dos Santos TB, de Souza SGH, Vieira LGE, Pereira LFP, Domingues DS (2013) Nitrogen starvation, salt and heat stress in coffee (Coffea arabica L.): Identification and validation of new genes for qPCR normalization. Mol Biotechnol 53:315-325

Denoeud F, Carretero-Paulet L, Dereeper A, Droc G, Guyot R, Pietrella M, Zheng C, Alberti A, Anthony F, Aprea G et al (2014) The coffee genome provides insight into the convergent evolution of caffeine biosynthesis. Science 345:1181-1184

Fernandes-Brum CN, Garcia B, Moreira R, Sagio SA (2017) A panel of the most suitable reference genes for RT-qPCR expression studies of coffee: screening their stability under different conditions. Tree Genet Genomes 13:131-144

Figueiredo A, Loureiro A, Batista D, Monteiro F, Várzea V, Pais MS, Gichuru EK, Silva MC (2013) Validation of reference genes for normalization of qPCR gene expression data from Coffea spp. hypocotyls inoculated with Colletotrichum kahawae. BMC Res Notes 6:388-398

Freitas NC, Barreto HG, Fernandes-Brum CN, Moreira RO, ChalfunJunior A, Palva LV (2017) Validation of reference genes for qPCR analysis of Coffea arabica L. somatic embryogenesis-related tissues. Plant Cell Tiss Organ Cult 128:663-678

Frischknecht PM, Ulmer-Dufek J, Baumann TW (1985) Purine alkaloids formation in buds and developing leaflets of Coffea arabica: expression of an optimal defence strategy? Phytochemistry 25:613-616 
Giridhar P, Kumar A, Simmi PS (2012) Differential expression of WRKY transcriptional factors in endosperm tissues during stress and ontogeny of fruits of Coffea canephora with respect to caffeine biosynthesis. In: Proceedings of 24th International Conference on Coffee Science (ASIC), Sanjose, Costa Rica. pp. 522-526

Goulao LF, Fortunato AS, Ramalho JC (2011) Selection of reference genes for normalizing quantitative Real-Time PCR gene expression data with multiple variables in Coffea spp. Plant Mol Biol Report 30:741-759

Joseph JT, Poolakkalody NJ, Shah JM (2018) Plant reference genes for development and stress response studies. J Biosci 43:173-187

Kato M, Mizuno K (2004) Caffeine synthase and related methyltransferases in Plants. Front Biosci 9:1833-1842

Kozera B, Rapacz M (2013) References genes in real-time PCR. J Appl Genet 54:391-406

Kumar A, Giridhar P (2015b) Salicylic acid and methyl jasmonate restore the transcription of caffeine biosynthetic $N$-methyltransferases from a transcription inhibition noticed during late endosperm maturation in coffee. Plant Gene 4:38-44

Kumar A, Simmi PS, Naik GK, Giridhar P (2015a) RP-HPLC and transcript profile indicate increased leaf caffeine in Coffea canephora plants by light. J Biol Earth Sci 5:1-9

Kumar A, Naik GK, Simmi PS, Giridhar P (2015c) Salinity and drought response alleviate caffeine content of young leaves of Coffea canephora var. Robusta cv. S274. J Appl Biol Biotechnol 3:50-60

Kumar A, Naik GK, Giridhar P (2017) Dataset on exogenous application of salicylic acid and methyl jasmonate and the accumulation of caffeine in young leaf tissues and catabolically inactive endosperms. Data Brief 13:22-27
Livak KJ, Schmittgen TD (2001) Analysis of relative gene expression data using real time quantitative PCR and the $2^{-\Delta \Delta C T}$ method. Methods 25:402-408

Perrois C, Strickler SR, Mathieu G, Lepelley M, Bedon L, Michaux S, Husson J, Mueller L, Privat I (2015) Differential regulation of caffeine metabolism in Coffea arabica (Arabica) and Coffea canephora (Robusta). Planta 241:179-191

Pfaffl MW, Tichopad A, Prgomet C, Neuvians TP (2004) Determination of stable housekeeping genes, differentially regulated target genes and sample integrity: BestKeeper-excel-based tool using pair-wise correlations. Biotechnol Lett 26:509-515

Pfaffl MW (2001) A new mathematical model for relative quantification in real-time RT-PCR. Nucl Acids Res 29:e45

Ruijter JM, Pfaffl MW, Zhao S, Spiess AN, Boggy G, Blom J, Rutledge RG, Sisti D, Lievens A, de Preter K (2012) Evaluation of qPCR curve analysis methods for reliable biomarker discovery: Bias, resolution, precision, and implications. Methods 59:32-46

Silver N, Best S, Jiang J, Thein SL (2006) Selection of housekeeping genes for gene expression studies in human reticulocytes using real-time PCR. BMC Mol Biol 7:33. https://doi. org/10.1186/1471-2199-7-33

Vandesompele J, De Preter K, Pattyn F, Poppe B, Van Roy N, De Paepe A, Speleman F (2002) Accurate normalization of real-time quantitative RT-PCR data by geometric averaging of multiple internal control genes. Genome Biol 3:34.1-34.11

Vieira A, Talhinas P, Loureiro A, Duplessis S, Fernandez D, Silva M, Paulo C, Azinheira OS (2011) Validation of RT-PCR reference genes for in planta expression studies in Hemileia vastatrix, the causal agent of coffee leaf rust. Fungal Biol 115:891-901 